\title{
ON THE LOCAL BOUNDEDNESS OF SINGULAR INTEGRAL OPERATORS
}

\author{
MARK LECKBAND
}

\begin{abstract}
The class of singular integral operators whose kernels satisfy the usual smoothness conditions is studied. Let such an operator be denoted by $K$. We establish necessary conditions that imply $K$ has local (weighted) $L^{p}$ norm inequalities.

The underlying principle is as follows. If $\chi_{Q}$ is the characteristic function of a fixed cube $Q$ of $R^{n}$, or all of $R^{n}$, then $K \chi_{Q}$ and (the adjoint of $K$ ) $K^{*} \chi_{Q}$ determine the boundedness properties of $K$ for functions supported in a proper fraction of $Q$.
\end{abstract}

In [6], David and Journé classified Calderón-Zygmund operators that map $L^{p}\left(R^{n}\right)$ boundedly into $L^{p}\left(R^{n}\right)$. What we propose to do is establish conditions that imply a singular integral operator $K$ has local (weighted) $L^{p}$ norm inequalities when $K$ is associated with a kernel $k$ that satisfies the usual smoothness conditions,

$$
\left|k(x, y)-k\left(x^{\prime}, y\right)\right|+\left|k(y, x)-k\left(y, x^{\prime}\right)\right| \leq C\left|x-x^{\prime}\right|^{\delta} /|x-y|^{n+\delta},
$$

for some $0<\delta<1$ and all $(x, y),\left(x^{\prime}, y\right)$ with $x \neq y$ and $\left|x-x^{\prime}\right| \leq \frac{1}{2}|x-y|$. The other condition is

$$
|k(x, y)| \leq C|x-y|^{-n} \text {. }
$$

Our approach will be similar to that of $[8]$.

Let $Q$ be a bounded cube of $R^{n}$ or all of $R^{n}$. Then how $K$ and the adjoint $K^{*}$ operate on the characteristic function of $Q$ will determine the local $L^{p}$ or weighted $L^{p}$ boundedness of $K$. Let $a Q$ be the cube $Q$ dilated by $a$ keeping the center the same. We define (Definition 4.0) a local weighted version of the weak boundedness property of $[6]$ which we call $\mathrm{WBP}_{0}(Q)$. This property is a necessary weakening of the w.b.p. The main result is the following theorem. The associated maximal operator of $K$ will be denoted by $K_{*}$.

THEOREM. Let $K$ satisfy $\mathrm{WBP}_{0}(Q)$ for a $p_{0}>2$. Then there is a positive constant $C_{p_{0}}<1 / 400$ such that for $f$ supported in $C_{p_{0}} Q$,

$$
\int\left[K_{*} f(x)\right]^{p} w_{p_{0}}^{p}(x) d x \leq A_{p_{0}, p} \int|f(x)|^{p} d x,
$$

and

where

$$
\int\left[K_{*} f(x)\right]^{p} d x \leq B_{p_{0}, p} \int|f(x)|^{p} w_{p_{0}}^{-p}(x) d x,
$$

$$
w_{p_{0}}(x)=\left[\sup _{x \in J} \frac{1}{|J|} \int_{J}\left\{\left|\beta_{L}^{\#}(s)\right|+\left|\beta_{M}^{\#}(s)\right|+1\right\}^{p_{0}} d s\right]^{-1 / p_{0}}
$$

Received by the editors June 1, 1986.

1980 Mathematics Subject Classification (1985 Revision). Primary 42B25. 
and $\beta_{L}(x)=X_{Q}(x) K\left[X_{Q}\right](x)-\chi_{Q}(x) \operatorname{ave}_{Q}\left(X_{Q} K\left[X_{Q}\right]\right)$ with $X_{Q}$ being the characteristic function of $Q$ with smooth edges when necessary. The function $\beta_{M}(x)$ is similarly defined using $K^{*}$.

If $Q$ is $R^{n}$ and $\beta_{L} 1=K 1, \beta_{M} 1=K^{*} 1$ both belong to BMO, then the above theorem is just the T1 theorem of David and Journé [6].

For an application of the above theorem, we look at a commutator with fractional differentiation defined in Murray [10] for functions $A \in \operatorname{Lip}_{\delta}\left(R^{n}\right) \backslash I_{\delta}(\mathrm{BMO}), 0<$ $\delta<1[\mathbf{1 4}]$. We list the result as Theorem 6.2 . Finally we would like to make two remarks.

The restriction of $p_{0}>2$ arises initially in Theorem 3.1. This restriction is most useful in establishing various (and seemingly unrelated to Theorem 3.1) facts throughout the later sections. However, it may be possible to improve Theorem 3.1 to be able to consider $1<p_{0} \leq 2$.

The second remark is that the smoothness conditions may be considerably weakened without changing the proofs and statements appreciably. For instance, one could only require

$$
\begin{aligned}
& \left|k(x, y)-k\left(x^{\prime}, y\right)\right| \leq \frac{C\left|x-x^{\prime}\right|^{\delta}}{|x-y|^{n+\delta}}\left(M_{|x-y|} \beta_{L}^{\#}(y)+1\right), \\
& \left|k(y, x)-k\left(y, x^{\prime}\right)\right| \leq \frac{C\left|x-x^{\prime}\right|^{\delta}}{|x-y|^{n+\delta}}\left(M_{|x-y|} \beta_{M}^{\#}(y)+1\right),
\end{aligned}
$$

and

We define

$$
|k(x, y)| \leq C \frac{1}{|x-y|^{n}}\left(M_{|x-y|}\left[\beta_{L}^{\#}+\beta_{M}^{\#}\right](y)+1\right) .
$$

$$
M_{t} f(y)=\sup _{y \in Q, s(Q)>5 t} \frac{1}{|Q|} \int_{Q}|f(x)| d x .
$$

The derived paraproducts $L$ and $M$ satisfy these conditions.

Finally, I would like to thank the referee for many helpful suggestions.

1. Preliminaries. The definitions and terminology we shall use will follow that of [ $\mathbf{7}$ and 8] with some necessary modifications. Most of the statements and proofs of lemmas and theorems are local; however it is to be noted that they are still true for all of $R^{n}$. Therefore, $Q$ will always be a bounded cube of $R^{n}$ centered at 0 , or all of $R^{n}$. We include the possibility of all of Euclidean space to be able to state simply global results and we center at 0 for ease of notation. We shall use $a Q$ to denote $Q$ dilated by $a$. We shall use $s(Q)$ and $d(Q)$ to denote the edge length and diameter of $Q$ respectively.

The operators we shall study will be associated to a kernel $k(x, y)$ which is defined on $R^{n} \times R^{n} \backslash \Delta, \Delta=\{(x, y): x \neq y\}$, and for which there are constants $C>0$ and $0<\delta<1$ such that

$$
\left|k(x, y)-k\left(x^{\prime}, y\right)\right|+\left|k(y, x)-k\left(y, x^{\prime}\right)\right| \leq \frac{C\left|x-x^{\prime}\right|^{\delta}}{|x-y|^{n+\delta}},
$$

for all $(x, y),\left(x^{\prime}, y\right) \in R^{n} \times R^{n} \backslash \Delta$ with $\left|x-x^{\prime}\right| \leq \frac{1}{2}|x-y|$. Also $k(x, y)$ will satisfy the usual growth condition

$$
|k(x, y)| \leq C|x-y|^{-n}, \quad \text { for } x \neq y .
$$


The operators $K$ that we will study will be continuous linear maps from the space of smooth functions with compact support, $C_{c}^{\infty}\left(R^{n}\right)$, to $\left[C_{c}^{\infty}\left(R^{n}\right)\right]^{\prime}$ having the property that

$$
\langle g, K f\rangle=\iint g(x) k(x, y) f(y) d y d x,
$$

for test functions $g$ and $f$ with disjoint support and $k(x, y)$ is a kernel satisfying (1.1) and (1.2).

Such operators have a natural extension to the space of bounded smooth functions by defining them as distributions of smooth functions with compact support and vanishing mean. We localize this well-known idea as follows. Let $\phi \geq 0$ be a $C_{c}^{\infty}\left(R^{n}\right)$ function, with $\int \phi=1$ and denote $\phi_{t}(x)=\phi(x / t) / t^{n}$. Then for smooth functions $g$ compactly supported in $\frac{1}{2} Q, C_{0}^{\infty}\left(\frac{1}{2} Q\right)$, we have $\left\langle g, K\left(\phi_{t} * X_{Q}\right)\right\rangle=$ $\langle g, K 1\rangle+\left\langle g, K\left(1-\phi_{t} * X_{Q}\right)\right\rangle$, where $K 1$ is the distribution found by applying $K$ to the constant function whose value is 1 , (see Lemma 1 of [8]). The inner product on the far left equals

$$
\iint g(x)\left[k(x, y)-k\left(x_{0}, y\right)\right]\left(1-\phi_{t}(y) * X_{Q}(y)\right) d y d x
$$

for $x_{0}$ in $\frac{1}{2} Q$ and small $t$. This integral is absolutely convergent uniformly for small $t$. Thus for $g$ in $C_{0}^{\infty}\left(\frac{1}{2} Q\right)$, we define

$$
\left\langle g, K X_{Q}\right\rangle=\lim _{t \rightarrow 0}\left\langle g, K\left(\phi_{t} * X_{Q}\right)\right\rangle .
$$

The above definition is easily seen to be independent of the $C_{c}^{\infty}\left(R^{n}\right)$ function $\phi$ that integrates to 1 by the above observations. We complete the preliminaries with the following elements of Littlewood-Paley theory.

Let $\psi$ always denote a radial $C^{\infty}$ function supported in $\{|x|<1\}$ such that

(i) $\psi(x)=0$ for $|x|<\frac{1}{2}$.

(ii) $\int \psi=0$.

(iii) $\int(\hat{\psi}(t))^{2} d t / t=1, \hat{\psi}$ is the Fourier transform of $\psi$.

Condition (i) will be very useful when we localize our Littlewood-Paley operators in the next section. The rest are standard conditions. Let $\psi_{t}(x)=\psi(x / t) / t^{n}$ and define $Q_{t} f(x)=\psi_{t} * f(x), f$ in $L^{p}, 1<p<\infty$. We will construct operators $g_{\psi}, S_{\psi}, \tilde{g}_{\delta}$ that are generalizations of the $g$-function Lusin area integral, and $g_{\lambda}^{*}$ functions of $[\mathbf{1 0}]$ using $\psi$ instead of the Poisson kernel.

The immediate facts we need are that $g_{\psi} f(x)=\left|\int\left[Q_{t} f(x)\right]^{2} d t / t\right|^{1 / 2}$ is $L^{p}$ norm equivalent to $f$ with equal $p=2$ norms, [7], and

$$
\begin{aligned}
\int g(x) f(x) d x & =\iint Q_{t}\left[Q_{t} g(x)\right] f(x) \frac{d t}{t} d x \\
& =\iint Q_{t} g(x) Q_{t} f(x) \frac{d t}{t} d x
\end{aligned}
$$

We will need one more operator, that being the approximation to the identity. Let $\rho$ denote a positive radial $C^{\infty}$ function supported in $\left\{\frac{1}{2}<|x|<1\right\}$ such that $\int \rho=1$. Let $\rho_{t}(x)$ be $\rho(x / t) / t^{n}$ and define $P_{t} f=\rho_{t} * f$. 
2. Localizing the $g_{\psi}$-function. Given a function $f$ supported in a cube $Q$, it is reasonable to expect that the range of integrations used in computing the $L^{p}$ norm of $g_{\psi} f$ can be restricted depending upon the dimensions of $Q$. This is the content of the next two lemmas which allow us to do all of our estimations within a multiple of $Q$. Before starting we would like to remark that a more natural approach to the theorems of this paper might be to regard $Q$ as $T^{n}$, the $n$ torus, and set up the Littlewood-Paley machinery using the pertinent facts of [13]. Unfortunately it seems the smoothness property $(1.1)$ is lost when periodizing $k(x, y)$.

In anticipation of the $A_{1}$ weight $w_{p_{0}}$ of Theorem 5.2, we consider $A_{p}$ weights [9] for Lemmas 2.1 and 2.2 .

LEMMA 2.1. Given $1<p<\infty, u \in A_{p}$, let $f \in L_{u}^{p}$ and be supported in cube $Q$. There is a constant $C_{p}$ such that

$$
\int|f(x)|^{p} u(x) d x \leq C_{p} \int_{C_{p} Q}\left|\int_{0}^{C_{p} s(Q)}\left(Q_{t} f(x)\right)^{2} \frac{d t}{t}\right|^{p / 2} u(x) d x .
$$

PROOF. The operator $g_{\psi} f=\left(\int\left(Q_{t} f\right)^{2} d t / t\right)^{1 / 2}$ is an isometry on $L^{2}$ and is bounded on $L_{u}^{p}$ for all $u \in A_{p}$ and $1<p<\infty$. Thus $g_{\psi} f$ and $f$ have equivalent weighted norms, [7, p. 79].

Assume $f \geq 0$ and let $C>3$; we will choose $C$ later. Then we have

$$
\begin{aligned}
\int f^{p}(x) u(x) d x \leq & C_{u} \int_{C Q}\left(g_{\psi} f(x)\right)^{p} u(x) d x \\
& +C_{u} \int_{R^{n} \backslash C Q}\left(g_{\psi} f(x)\right)^{p} u(x) d x
\end{aligned}
$$

We use property (i) of $\psi$ to get

$$
\int\left(g_{\psi} f(x)\right)^{p} u(x) d x=\int_{C Q}\left|\int_{0}^{2 C d(Q)}\left(Q_{t} f(x)\right)^{2} \frac{d t}{t}\right|^{p / 2} u(x) d x .
$$

The choice of $C$ comes in with estimating the second integral on the right side of (2.2). Simply choose the constant large enough so that this term is at most half the value of the integral on the left of (2.2). To see that this can always be done, observe $Q_{t} f(x)=0$ for $x \in R^{n} \backslash C Q$ and $0<t<|x| / 2$. Thus, for $x \in R^{n} \backslash C Q$,

$$
\left(g_{\psi} f(x)\right)^{p} \leq D_{p}\left|\int_{Q} f\right|^{p} \cdot|x|^{-p n}
$$

and using Hölder's inequality we have the above is less than

$$
D_{p}\left|\int_{Q} f^{p}(y) u(y) d y\right| \cdot\left|\int_{Q} u^{1-p^{\prime}}(y) d y\right|^{p-1} \cdot|x|^{-p n} .
$$

Note that $D_{p}$ depends upon $p$ and $\|\psi\|_{\infty}$. Since $u \in A_{p}$ we have $u^{1-p^{\prime}} \in A_{p^{\prime}}$, in particular $A_{\infty}$. So for some constant $E_{C}$ greater than a positive power of $C$, we have for $x \in R^{n} \backslash C Q$,

$$
\left|\int_{Q} u^{1-p^{\prime}}(y) d y\right|^{p}|x|^{-p n} \leq \frac{A}{E_{C}}\left|M\left[\chi_{C Q} u^{1-p^{\prime}}\right](x)\right|^{p},
$$


where $M$ is the Hardy-Littlewood maximal operator. Thus

$$
\begin{aligned}
\int_{R^{n} \backslash C Q}\left(g_{\psi} f(x)\right)^{p} u(x) d x & \leq \frac{A^{\prime}}{E_{C}^{1 / p^{\prime}}} \int_{Q} f^{p}(x) u(x) d x\left|\int_{C Q} u^{1-p^{\prime}}(x) d x\right|^{-1} \\
& \cdot \int M^{p}\left[\chi_{C Q} u^{1-p^{\prime}}\right](x) u(x) d x \\
& \leq \frac{A^{\prime \prime}}{E_{C}^{1 / p^{\prime}}} \int_{Q} f^{p}(x) u(x) d x
\end{aligned}
$$

The proof is completed by choosing $C$ large enough so that the constant $A^{\prime \prime} / E_{C}^{1 / p^{\prime}}$ is less than $\frac{1}{2} C_{u}$.

LEMMA 2.2. Given $1<p<\infty, u \in A_{p}$, and $f$ in $C^{\infty}$ supported in $Q$, suppose $K f \in L_{u}^{p}(4 Q)$. Then there is a constant $B_{p}$ such that

$$
\begin{aligned}
\int_{4 Q}( & K f(x))^{p} u(x) d x \\
\leq & B_{p} \int_{B_{p} Q}\left|\int_{0}^{B_{p} s(Q)}\left\{\iint Q_{t} K_{1} Q_{t^{\prime}}(x, y) Q_{t^{\prime}} f(y) \frac{d t^{\prime}}{t^{\prime}} d y\right\}^{2} \frac{d t}{t}\right|^{p / 2} u(x) d x \\
& +B_{p} \int f^{p}(x) u(x) d x,
\end{aligned}
$$

where $k_{1}(x, y)=X_{Q_{0}}(x) X_{Q_{0}}(y) \cdot k(x, y), Q_{0}=100 B_{p} Q$.

Proof. By Lemma 2.1 there is a constant $C$ such that

$$
\begin{aligned}
\int_{4 Q}( & K f(x))^{p} u(x) d x \\
\leq & 2 C_{p} \int_{C_{p} Q}\left|\int_{0}^{C_{p} s(Q)} Q_{t}\left(X_{Q_{0}} K f\right)(x) \frac{d t}{t}\right|^{p / 2} u(x) d x \\
& +2 C_{p} \int_{C_{p} Q}\left|\int_{0}^{C_{p} s(Q)} Q_{t}^{2}\left(X_{Q_{0} \backslash 4 Q} K f\right)(x) \frac{d t}{t}\right|^{p / 2} u(x) d x,
\end{aligned}
$$

where $Q_{0}=400 C_{p} Q$. The first integral is precisely the first integral of our conclusion if we let $B_{p}=4 C_{p}$.

The second integral is bounded by $2 C_{p} \int_{Q_{0} \backslash 4 Q}(K f)^{p}(x) u(x) d x$. For $x \in Q_{0} \backslash 4 Q$ and $y \in Q$, we have $|k(x, y)| \sim|x-y|^{-n}<C|Q|$. Thus we conclude the proof with

$$
\begin{aligned}
2 C_{p} \int_{Q_{0} \backslash 4 Q}(K f)^{p}(x) u(x) d x & \leq C \int M^{p}(f)(x) u(x) d x \\
& \leq C^{\prime} \int|f(x)|^{p} u(x) d x .
\end{aligned}
$$

We would like to remark that the constant $100 B_{p}$ is at least 400 .

Lemma 2.2 sets the stage where the estimations to be done take place. The first integral on the right of $(2.3)$ contains the quantity $Q_{t} K_{1} Q_{t^{\prime}}(x, y)$, and the kernel of 
$K_{1}$ is $k(x, y) X_{Q_{0}}(x) X_{Q_{0}}(y)$. The function $X_{Q}(y)$ is a $C^{\infty}$ function, where we have assumed a smoothing of the edges. This will not affect the estimates we shall do.

Note that the ranges of integration for the variables $y$ and $t^{\prime}$ cannot be restricted; however those ranges of the variables $x$ and $t$ have been sufficiently restricted in order to do our estimations locally.

3. The operators $L$ and $M$. We shall decompose the operator $K_{1}$ of Lemma 2.2 as the sum of three operators $K_{0}, L$ and $M$, where $K_{0}=K_{1}-L-M$. We define the operators $L$ and $M$ below as in [8]. However, since we are not assuming $K$ and its adjoint necessarily map 1 into BMO, the properties of $L$ and $M$ must be established anew. In particular, we will establish their behavior as maps of $L^{p}$ (Theorem 3.1), and the smoothness properties of their kernels (Lemma 4.1 in $\S 4$ ).

In the sense of (1.4) with $Q$ replaced by $Q_{0}$, we desire to have $L 1=K_{1} 1, M 1=0$, $K_{0} 1=0$, and the adjoint operators satisfy $L^{*} 1=0, M^{*} 1=K^{*} 1$, and $K^{*} 1=0$. The special property of $K_{0}$ and its adjoint mapping 1 into 0 will allow us to obtain pointwise estimates on $\left|Q_{t} K_{0} Q_{t},(x, y)\right|$ (Proposition 4.3) that are weighted variants of those in [8]. This is our reason for the decomposition using $L$ and $M$.

As was mentioned in the last section, a smoothing of the edges of $Q_{0}$ shall be done. For example, consider $P_{\gamma} \chi_{Q_{0}}, 0<\gamma<\frac{1}{4}$. Fix $\gamma$ and note that no estimates will depend upon $\gamma$. We use $X_{Q_{0}}$ to denote $P_{\gamma} \chi_{Q_{0}}$.

Let

$$
\beta_{L}(x)=X_{Q_{0}}(x) K_{1} 1(x)=X_{Q_{0}}(x) \int k(x, y) X_{Q_{0}}(y) d y,
$$

and $\beta_{M}(x)=X_{Q_{0}}(x) K_{1}^{*} 1(x)$, and we require that their averages over $Q_{0}$ vanish. To ensure this happens for finite $Q_{0}$, redefine $\beta_{L}$ to be $\beta_{L}-\chi_{Q_{0}}$ ave $_{Q_{0}} \beta_{L}$, and similarly for $\beta_{M}$. Let

$$
\begin{aligned}
L f(x) & =\int Q_{t}\left[Q_{t} \beta_{L} P_{t} f\right](x) \frac{d t}{t}, \\
M^{*} f(x) & =\int Q_{t}\left[Q_{t} \beta_{M} P_{t} f\right](x) \frac{d t}{t},
\end{aligned}
$$

and for $f, g \in C_{c}^{\infty}$,

$$
\begin{gathered}
\langle g, L f\rangle=\iint Q_{t} g(x) Q_{t} \beta_{L}(x) P_{t} f(x) \frac{d t}{t} d x \\
\langle g, M f\rangle=\left\langle M^{*} g, f\right\rangle=\iint P_{t} g(x) Q_{t} \beta(x) Q_{t} f(x) \frac{d t}{t} d x .
\end{gathered}
$$

The estimates and norm inequalities that will be satisfied have a weight derived from the functions $\beta_{L}$ and $\beta_{M}$. We define next the $p$-sharp maximal function of $\beta_{L}$ and $\beta_{M}$ that will be the weight of our results.

DEFINITION 3.0. Given $Q_{0}$ and $p_{0} \geq 1$, let

$$
w_{p_{0}}(x)=\left[\sup _{x \in J} \frac{1}{|J|} \int_{J}\left|\beta_{L}^{\#}(y)+\beta_{M}^{\#}(y)+1\right|^{p_{0}} d y\right]^{-1 / p_{0}}
$$

where

$$
\beta^{\#}(y)=\sup _{y \in Q}\left[\frac{1}{|Q|} \int_{Q}\left|\beta(s)-\operatorname{ave}_{Q} \beta\right|^{p_{0}} d s\right]^{1 / p_{0}} .
$$

Note that we are also using $J$ to denote a cube of $R^{n}$. 
THEOREM 3.1. Given $p_{0}>2$ we have

$$
L, M^{*}: L^{p} \rightarrow L_{w_{p_{0}}^{p}}^{p}, \quad \text { for } 2 \leq p \leq q\left(p_{0}\right)
$$

and

$$
L, M, L^{*}, M^{*}: L_{w_{p_{0}}^{-2}}^{2} \rightarrow L^{2} .
$$

The norm constants for the above maps only depend upon the dimension $n$ and $p_{0}$.

PROOF. We shall just prove (3.2) for the operator $L$ since the argument for $M^{*}$ is similar.

Fix $p_{0}>2$ and $w=w_{p_{0}}^{2}$. We will first show that the operator $L_{w}$, defined by

$$
L_{w} f(x)=\left|\int P_{t}^{2} f(x) Q_{t}^{2} \beta_{L}(x) w(x) \frac{d t}{t}\right|^{1 / 2}
$$

maps $L^{p}$ into $L^{p}, 2 \leq p \leq q\left(p_{0}\right)$, boundedly with a constant that depends only on dimension $n$ and $p_{0}$.

To begin, we observe that $w^{-1} \in A_{1}$ since $p_{0}>2$, and $w \in A_{2}$ with $w u \in A_{2}$ for any $u \in A_{1}[\mathbf{5}]$. By a method of de Francia [11], to show an operator maps $L^{p}$ boundedly into $L^{p}$, one need only show $L_{w}$ maps $L_{u}^{2}$ into $L_{u}^{2}$ uniformly for $u \in A_{1}$ with uniformly bounded $A_{1}$ constants. We will only be able to show this for $u$ with $A_{1}$ constants sufficiently close to 1 . This restriction puts an upper bound on $p$ which we denote by $q\left(p_{0}\right)$.

Now to show $L_{w}$ maps $L_{u}^{2}$ into $L_{u}^{2}$ boundedly, we only need to show that $\left|Q_{t}^{2} \beta_{L}(x) w(x)\right|(d t / t) u(x) d x$ is a Carleson measure [12] with respect to $u \in A_{1}$. This implies the sequence of inequalities;

$$
\begin{aligned}
\iint P_{t}^{2} f(y) Q_{t}^{2} \beta_{L}(y) w(y) \frac{d t}{t} u(y) d y \\
\quad \leq \int M^{2} f(y) u(y) d y \leq C \int|f(y)|^{2} u(y) d y .
\end{aligned}
$$

Let $Q \subset R^{n}$, and let $\beta_{1}=\beta_{L}-(1 /|3 Q|) \int_{3 Q} \beta_{L}(x) d x$ and $\beta_{2}=\beta_{1} \chi_{3 Q}$. Then

$$
\begin{aligned}
& \int_{Q} \int_{0}^{s(Q)} Q_{t}^{2} \beta_{L}(y) \frac{d t}{t} w(y) u(y) d y=\int_{Q} \int_{0}^{s(Q)} Q_{t}^{2} \beta_{1}(y) \frac{d t}{t} w(y) u(y) d y \\
& \quad \leq \iint Q_{t}^{2} \beta_{2}(y) \frac{d t}{t} w(y) u(y) d y \leq C \int \beta_{2}^{2}(y) w(y) u(y) d y \\
& \quad \leq C \underset{\underline{y} \in 3 Q}{\operatorname{ess} \sup } w(\underline{y})\left[\int_{3 Q}\left|\beta_{1}(y)\right|^{2(1+\varepsilon) / \varepsilon} d y\right]^{1 /(1+\varepsilon)}\left|\int_{3 Q} u^{1+\varepsilon}(y) d y\right|^{1 /(1+\varepsilon)} .
\end{aligned}
$$

The above is less than $C u(Q)$ if we require $\varepsilon$ to be such that $2(1+\varepsilon) / \varepsilon=p_{0}$ and $u$ to satisfy the reverse Hölder condition for such an $\varepsilon$. Therefore the $A_{1}$ constant of $u$ must be sufficiently close to 1 and so $2 \leq p \leq q\left(p_{0}\right)$. Thus we have $L_{w}$ is a bounded map of $L^{p}$. 
Now we choose a $g \in L_{w_{p_{0}}^{p}}^{p^{\prime}}$ with $\|g\|_{p^{\prime}, w_{p_{0}}^{p}} \leq 1$. Then for $f \in L^{p}$ and $p \geq 2$,

$$
\begin{aligned}
\left|\langle g, L f\rangle_{w_{p_{0}}^{p}}\right|= & \left|\iint Q_{t}\left(g w_{p_{0}}^{p}\right)(y) Q_{t} \beta_{L}(y) P_{t} f(y) \frac{d t}{t} d y\right| \\
\leq & \left.\left.\left|\int\right| \int Q_{t}^{2}\left(g w_{p_{0}}^{p}\right)(y) \frac{d t}{t}\right|^{p^{\prime} / 2} w_{p_{0}}^{-p^{\prime}}(y) d y\right|^{1 / p} \\
& \left.\left.\cdot\left|\int\right| \int Q_{t}^{2} \beta_{L}(y) P_{t}^{2} f(y) w(y) \frac{d t}{t}\right|^{p / 2} d y\right|^{1 / p}
\end{aligned}
$$

by a double application of Hölder's inequality. Since $p \geq 2$ we have $p^{\prime} / p_{0}<1$ and $w_{p_{0}}^{-p^{\prime}} \in A_{1}$, so the above reduces to $C\|g\|_{p^{\prime}, w_{p_{0}}^{p}}\left\|L_{w} f\right\|_{p} \leq C\|f\|_{p}$.

To show (3.3) we let $g \in L^{2}$ with $\|g\|_{2}=1$. Then

$$
|\langle g, L f\rangle| \leq\left|\iint Q_{t}^{2} g(x) \frac{d t}{t} d x\right|^{1 / 2} \cdot\left|\iint Q_{t}^{2} \beta_{L}(x) P_{t}^{2} f(x) \frac{d t}{t} d x\right|^{1 / 2},
$$

again by a double application of Hölder's inequality. The second factor is just $\left\|L_{w} f\right\|_{2, w_{p_{0}}^{-2}} \leq C\|f\|_{2, w_{p_{0}}^{-2}}$. To finish we observe that $L$ and its adjoint share the same $p=2$ norm.

We will complete this section with one more result, that is $L 1=\beta, L^{*} 1=0$, etc. in a sense akin to Lemma 1 of [8].

LEMMA 3.2. Let $g \in C_{0}^{\infty}$ supported in $\frac{1}{2} Q_{0}$. Let $\phi \in C^{\infty}$ such that $\phi=1$ on $\left\{|z| \leq \frac{1}{2}\right\}$ and $\operatorname{supp} \phi$ is contained in $\{|z|<1\}$. Then if $\phi^{t}(x)=\phi(x / t)$,

$$
\begin{gathered}
\lim _{t \rightarrow \infty}\left\langle g, L \phi^{t}\right\rangle=\left\langle g, \beta_{L}\right\rangle, \\
\lim _{t \rightarrow \infty}\left\langle g, M^{*} \phi^{t}\right\rangle=\left\langle g, \beta_{M}\right\rangle,
\end{gathered}
$$

(3.6) $\lim _{t \rightarrow \infty}\left\langle g, K_{0} \phi^{t}\right\rangle, \quad \lim _{t \rightarrow \infty}\left\langle g, K_{0}^{*} \phi^{t}\right\rangle, \quad \lim _{t \rightarrow \infty}\left\langle g, L^{*} \phi^{t}\right\rangle$, and $\lim _{t \rightarrow \infty}\left\langle g, M \phi^{t}\right\rangle=0$.

ProOF. We first consider the operator $L$. Then we have

$$
\begin{aligned}
\left|\left\langle g, L \phi^{t^{\prime}}\right\rangle\right| & =\left|\iint Q_{t}(g)(y) Q_{t} \beta_{L}(y) P_{t}\left(\phi^{t^{\prime}}\right)(y) \frac{d t}{t} d y\right| \\
& \leq \iint\left|Q_{t} g(y) \| Q_{t} \beta_{L}(y)\right| \frac{d t}{t} d y \\
& \leq\left.\left. C\|g\|_{p_{0}^{\prime}} \cdot\left|\int\right| \int Q_{t}^{2} \beta_{L}(y) \frac{d t}{t}\right|^{p_{0} / 2} d y\right|^{1 / p_{0}},
\end{aligned}
$$

by a double application of Hölder's inequality. The second integral can be shown to be less than $C \int_{Q_{0}}\left(\beta_{L}^{\#}\right)^{p_{0}}(x) d x$ since the support of $\beta_{L}$ is in $Q_{0}$ and ave $Q_{0} \beta_{L}=0$. Our weight is assumed to be not identically zero; thus the above is finite, allowing us to use the Lebesgue dominated convergence theorem, so

$$
\begin{aligned}
\lim _{t \rightarrow \infty}\left\langle g, L \phi^{t^{\prime}}\right\rangle & =\iint Q_{t} g(y) Q_{t} \beta_{L}(y) \frac{d t}{t} d y \\
& =\left\langle g(y), \beta_{L}(y)\right\rangle .
\end{aligned}
$$


To show $\lim \left\langle g, L^{*} \phi^{t^{\prime}}\right\rangle=0$, we observe that $t^{\prime} \gg 2 B_{p} Q, \operatorname{supp}(\psi) \cup \operatorname{supp}(\rho) \subset$ $\left\{\frac{1}{2}<|x|<1\right\}$ and $\operatorname{supp}(g) \subset 2 B_{p} Q$ implies

$$
\begin{aligned}
\left|\left\langle g, L^{*} \phi^{t^{\prime}}\right\rangle\right| & \leq \int_{R^{n} \backslash C_{1} Q} \int_{C_{2}|y|}^{3 C_{2}|y|}\left|Q_{t} \beta_{L}(y)\right|\left|P_{t} g(y)\right| \frac{d t}{t} d y \\
& \leq C_{3} \int\left|M\left(\beta_{L}\right)(y)\right||M g(y)| d y
\end{aligned}
$$

Now the argument is similar as before. That is, $\lim _{t^{\prime} \rightarrow \infty} Q_{t}\left(\phi^{t^{\prime}}\right)(y)=0$ and the Lebesgue dominated convergence theorem imply $\lim _{t^{\prime} \rightarrow \infty}\left\langle g, L^{*} \phi^{t^{\prime}}\right\rangle=0$. Note that the arguments for $M$ and $M^{*}$ are the same. We complete the proof by observing

$$
\lim _{t^{\prime} \rightarrow \infty}\left\langle g, K_{0} \phi^{t^{\prime}}\right\rangle=\lim _{t^{\prime} \rightarrow \infty}\left[\left\langle g, K_{1} \phi^{t^{\prime}}\right\rangle-\left\langle g, L \phi^{t^{\prime}}\right\rangle-\left\langle g, M \phi^{t^{\prime}}\right\rangle\right]=0,
$$

and the proof for $K_{0}^{*}$ is the same.

4. $\mathrm{WBP}_{0}(Q)$ and pointwise estimates. The weak boundedness property, w.b.p., of [8] is satisfied by operators with asymmetric kernels. We cannot always hope this to be the case, and a weighted variation, $\mathrm{WBP}_{0}(Q)$, is defined below in Definition 4.0. We will show that the operators $L, M$, etc. satisfy $\mathrm{WBP}_{0}\left(Q_{0}\right)$.

The main objective of this section is to derive a pointwise estimate on $\left|Q_{t} K_{0} Q_{t^{\prime}}(x, y)\right|$, Proposition 4.3. To do this we will need some preliminary results on the smoothness properties of the kernels of $L$ and $M$, and to show $K_{0}$ satisfies $\mathrm{WBP}_{0}\left(Q_{0}\right)$.

DEFINITION 4.0. The $\mathrm{WBP}_{0}(Q)$ property. Let $Q$ be a bounded cube of $R^{n}$, or all of $R^{n}$, and $p_{0} \geq 1$. Let $\xi$ and $\eta$ be $C^{\infty}$ functions supported in $\{|z|<40\}$, and suppose one integrates to 0 . Then $K$ will satisfy $\mathrm{WBP}_{0}(Q)$ if for $x \in Q$, and $0<t<(1 /(100)) \cdot s(Q)$, we have

$$
\left|\left\langle\xi_{t}^{x}, K \eta_{t}^{y}\right\rangle\right| \leq C_{p_{0}} / t^{n} \cdot w_{p_{0}}^{-1}(y),
$$

for $|x-y|<5 t$ and $w_{p_{0}}$ defined in Definition 3.0 using $Q$ instead of $Q_{0}$.

LEMMA 4.1. For $p_{0}>2$ we have the following smoothness estimates on the kernels of $L$ and $M$. Define

$$
M_{|u-v|} \beta^{\#}(v)=\sup _{v \in J, s(J)>5|u-v|} \frac{1}{|J|} \int_{J}\left|\beta_{L}^{\#}(x)\right|+\left|\beta_{M}^{\#}(x)\right| d x .
$$

Then

$$
\begin{gathered}
\int_{0}^{\infty}\left|L_{t}(u, v)-L_{t}(s, v)\right|+\left|L_{t}(v, u)-L_{t}(v, s)\right| \frac{d t}{t} \\
\quad+\int_{0}^{\infty}\left|M_{t}(u, v)-M_{t}(s, v)\right|+\left|M_{t}(v, u)-M_{t}(v, s)\right| \frac{d t}{t} \\
\leq \frac{C|u-s|}{|u-v|^{n+1}} M_{|u-v|} \beta^{\#}(v), \quad \text { for }|u-s|<\frac{1}{2}|u-v| .
\end{gathered}
$$

ProOF. We shall just work with $L$. For $t<\frac{1}{2}|u-v|$ we have $L_{t}(u, v)=$ $\int \psi_{t}(u-x)\left(Q_{t} \beta_{L}\right)(x) \rho_{t}(x-v) d x=0$. Hence the range of integration is over 
$t>\frac{1}{2}(|u-v| \wedge|s-v|)$. Since $|u-s|<\frac{1}{2}|u-v|$, we have $|s-v|$ is comparable to $|u-v|$. Moreover, observe that $\left|\psi_{t}(u-x)-\psi_{t}(s-x)\right| \leq C|u-s| / t^{n+1}$, and so

$$
\int_{0}^{\infty}\left|L_{t}(u, v)-L_{t}(s, v)\right| \frac{d t}{t} \leq C \int_{C^{\prime}|u-v|}^{\infty} \int \frac{|u-s|}{t^{n+1}}\left|Q_{t} \beta_{L}(x)\right| \rho_{t}(v-x) d x \frac{d t}{t} .
$$

For each $t, Q_{t}$ operating on a constant is 0 . Thus we have the above is less than or equal to

$$
|u-s| \int_{C^{\prime}|u-v|}^{\infty} \int \frac{1}{t^{n+1}} M_{t} \beta^{\#}(v) \rho_{t}(v-x) d x \frac{d t}{t} \leq C \frac{|u-s|}{|u-v|^{n+1}} M_{|u-v|} \beta^{\#}(v) .
$$

An important class of operators has kernels $k(x, y)$ that satisfy $(1.1),(1.2)$ and have $k(x, y)=-k(y, x)$. This class contains the commutators of Calderón for instance, and in [6] it is shown that this class satisfies the weak boundedness property or (4.1) with the weight replaced by a constant. Hence the hypothesis of the next lemma is satisfied for this class.

LEMMA 4.2. Assume $K_{1}$ satisfies the $\mathrm{WBP}_{0}\left(Q_{0}\right)$ for some $p_{0}>2$. Then $K_{0}, L$, and $M$ satisfy the same property for the same $p_{0}$.

PROOF. We only need to prove the lemma for $L$ and $M$, and since the proofs are similar we shall just prove the lemma for $L$.

By (3.3) we have for $p_{1}=\left(p_{0}+2\right) / 2>2$,

$$
\left|\left\langle\xi_{t}^{x}, L \eta_{t}^{y}\right\rangle\right| \leq \frac{C_{p_{1}}}{t^{n / 2}}\left|\int\right| \eta_{t}^{y}(z)\left|w_{p_{1}}^{-2}(z) d z\right|^{1 / 2} \leq \frac{C}{t^{n}}\left|\frac{1}{B_{t}(y)} \int_{B_{t}(y)} w_{p_{1}}^{-2}(z) d z\right|^{1 / 2} .
$$

Now let $\chi_{1}=\chi_{2 B_{t}(y)}$, and $\chi_{2}=\chi_{R^{n} \backslash 2 B_{t}(y)}$. Then

$$
\left|\frac{1}{B_{t}(y)} \int_{B_{t}(y)}\left[M\left(X_{1}\left[\left|\beta_{L}^{\#}\right|+\left|\beta_{M}^{\#}\right|+1\right]^{p_{1}}\right)(u)\right]^{2 / p_{1}} d u\right|^{1 / 2} \leq C_{p_{0}} w_{p_{0}}^{-1}(y)
$$

by an application of Hölder's inequality and $\left\|M\left[f^{p_{1}}\right]\right\|_{p_{0} / p_{1}}^{1 / p_{1}} \leq C\left\|f^{p_{0}}\right\|_{1}^{1 / p_{0}}$ for $p_{0}>$ $p_{1}>2$. Observe for $u \in B_{t}(y)$ that $M\left[\chi_{2} \cdot\left(\left|\beta_{L}^{\#}\right|+\left|\beta_{M}^{\#}\right|+1\right)^{p_{1}}\right]^{1 / p_{1(u)}}$ is basically constant; hence

$$
\left|\left\langle\xi_{t}^{x}, L \eta_{t}^{y}\right\rangle\right| \leq C_{p_{0}} w_{p_{0}}^{-1}(y) t^{-n}
$$

Before continuing we should remark that nowhere did we explicitly use the fact that $\xi$ or $\eta$ integrates to zero. This is because we only concerned ourselves with $|x-y|<5 t$, yet we still require this in our definition so as to include the aforementioned operators with asymmetric kernels, etc.

Recall that (2.3) of Lemma 2.2 set the stage where the estimations are to be done. Having $K_{1}=K_{0}+L+M$, and Theorem 3.1, we derive pointwise estimates on $\left|Q_{t} K_{0} Q_{t^{\prime}}(x, y)\right|$ in the same manner as is done in [8]. The proof is similar and the only thing one must do is take care with the weights and so forth involved.

PROPOSITION 4.3. Let $Q$ be contained in $R^{n}$, or be all of $R^{n}$, and $0<t<$ $B_{p} s(Q)$, where $B_{p}$ is the constant of Lemma 2.2. We have the following estimates on $K_{0}=K_{1}-L-M$.

$$
\begin{aligned}
& \left|Q_{t} K_{0} Q_{t^{\prime}}(x, y)\right| \leq C w_{p_{0}}^{-1}(y)\left(t^{\prime}\right)^{\delta} /\left[|x-y|^{n+\delta}+t^{n+\delta}\right], \\
& \quad \text { for } 0<t^{\prime}<t,|x-y|<5 t .
\end{aligned}
$$




$$
\begin{aligned}
& \left|Q_{t} K_{0} Q_{t^{\prime}}(x, y)\right| \leq C w_{p_{0}}^{-1}(x)(t)^{\delta} /\left[|x-y|^{n+\delta}+\left(t^{\prime}\right)^{n+\delta}\right], \\
& \text { for } 0<t<t^{\prime},|x-y|<5 t^{\prime} .
\end{aligned}
$$

$$
\begin{aligned}
& \left|Q_{t} K Q_{t^{\prime}}(x, y)\right| \leq C w_{p_{0}}^{-1}(x)\left(t \wedge t^{\prime}\right)^{\delta} /\left[|x-y|^{n+\delta}+\left(t \vee t^{\prime}\right)^{n+\delta}\right], \\
& \quad \text { for }|x-y|>5 t \vee t^{\prime} .
\end{aligned}
$$

PROOF. We will first consider the case $|x-y|>5 t \vee t^{\prime}$ and assume $0<t<t^{\prime}$. If $0<t^{\prime}<t$ then (2.3) implies $t^{\prime}$ is less than $B_{p} s(Q)$ and the argument is similar. Thus

$$
\left|Q_{t} K_{0} Q_{t^{\prime}}(x, y)\right| \leq\left|\left\langle\psi_{t}^{x}, K_{1} \psi_{t^{\prime}}^{y}\right\rangle\right|+\left|\left\langle\psi_{t}^{x}, L \psi_{t^{\prime}}^{y}\right\rangle\right|+\left|\left\langle\psi_{t}^{x}, M \psi_{t^{\prime}}^{y}\right\rangle\right| .
$$

Using the smoothness property (1.1) of $K$ and the mean 0 property of $\psi$, one derives $\left|\left\langle\psi_{t^{\prime}}^{x} K_{1} \psi_{t^{\prime}}^{y}\right\rangle\right| \leq C(t)^{\delta} /|x-y|^{n+\delta}$ the usual way. For the other two terms, we use the smoothness property (4.2) of $L$ and $M$ and the mean 0 property of $\psi$ to derive (just consider $L$ )

$$
\begin{aligned}
\left|\left\langle\psi_{t}^{x}, L \psi_{t^{\prime}}^{y}\right\rangle\right| & \leq\left|\int \psi_{t}^{x}(u) \int L_{t}(u, v)-L_{t}(x, v) \frac{d t}{t} \psi_{t^{\prime}}^{y}(v) d u d v\right| \\
& \leq C \int\left|\psi_{t}^{x}(u)\right| \frac{|u-x|}{|x-v|^{n+1}} M_{|u-v|} \beta^{\#}(v)\left|\psi_{t^{\prime}}^{y}(v)\right| d u d v \\
& \leq C \frac{(t)}{|x-y|^{n+1}} w_{p_{0}}^{-1}(x),
\end{aligned}
$$

since

$$
M_{|u-v|} \beta^{\#}(v) \leq C w_{p_{0}}(x), \quad \text { for }|u-x| \leq t .
$$

Finally we note that for $0<t^{\prime}<t$ the upper bounds derived are the same with $t$ replaced by $t^{\prime}$ and $w_{p_{0}}^{-1}$ may still be considered as a function of $x$. This establishes (4.5).

Now we consider $|x-y|<5 t \vee t^{\prime}$ and we assume $0<t<t^{\prime}$. Again the case for $0<t^{\prime}<t$ is similar. We begin as is done in [8] by letting $\alpha$ be a radial function equal to 1 on $\{|z|<20\}$ and 0 on $\{|z|>30\}$. Denote $\alpha^{x, t}(z)=\alpha((z-x) / t)$. Since $|x-y|<5 t^{\prime}$ we have $\alpha^{x, t^{\prime}}=1$ on $\operatorname{supp} \psi_{t^{\prime}}^{y}$. We require the following decomposition.

$$
\begin{aligned}
\left|Q_{t} K_{0} Q_{t}(x, y)\right| \leq & \left|\left\langle\psi_{t}^{x}, K_{0} \alpha^{x, t^{\prime}} \psi_{t^{\prime}}^{y}(x)\right\rangle\right| \\
& +\left|\left\langle\psi_{t}^{x}, K_{0}\left(\alpha^{x, t^{\prime}}-\alpha^{x, 2 t}\right)\left(\psi_{t^{\prime}}^{y}-\psi_{t^{\prime}}^{y}(x)\right)\right\rangle\right| \\
& +\left|\left\langle\psi_{t}^{x}, K_{0}\left(\psi_{t}^{y}-\psi_{t}^{y}(x)\right) \alpha^{x, 2 t}\right\rangle\right|
\end{aligned}
$$

We shall estimate the quantities on the right in order.

(I) Having $\lim _{\bar{t} \rightarrow \infty}\left\langle\psi_{t}^{x}, K_{1} \phi^{\bar{t}}\right\rangle=0$ from Lemma 3.2 , for ease of notation we write $\phi^{\bar{t}} \equiv 1$. The following estimates are uniform for all large $\bar{t}$.

$$
\begin{aligned}
& \left|\left\langle\psi_{t}^{x}, K_{0} \alpha^{x, t^{\prime}} \psi_{t^{\prime}}^{y}(x)\right\rangle\right|=\left|\left\langle\psi_{t}^{x}, K_{0}\left(\alpha^{x, t^{\prime}}-1\right) \psi_{t^{\prime}}^{y}(x)\right\rangle\right| \\
& \quad \leq C\left[\left|\left\langle\psi_{t}^{x}, K_{1}\left(\alpha^{x, t^{\prime}}-1\right)\right\rangle\right|+\left|\left\langle\psi_{t}^{x}, L\left(\alpha^{x, t^{\prime}}-1\right)\right\rangle\right|+\left|\left\langle\psi_{t}^{x}, M\left(\alpha^{x, t^{\prime}}-1\right)\right\rangle\right| \mid t^{-n},\right.
\end{aligned}
$$

since $\left\|\psi_{t^{\prime}}\right\|_{\infty} \leq C \cdot t^{\prime-n}$.

Given $u \in \operatorname{supp} \Gamma^{x}$ and $v \in \operatorname{supp}\left(\alpha^{x, t^{\prime}}-1\right)$, we have $|x-u|<\frac{1}{2}|u-v|$; hence the smoothness property (1.1) of $K$ and that of $L$ and $M(4.2)$ apply. The argument is now the same as before in the case $|x-y|>5 t \vee t^{\prime}$. Thus

$$
\left|\left\langle\psi_{t}^{x}, K_{0} \alpha^{x, t^{\prime}} \psi_{t^{\prime}}^{y}(x)\right\rangle\right| \leq \frac{C(t)^{\delta}}{\left(t^{\prime}\right)^{n+\delta}} w_{p_{0}}^{-1}(x)
$$


(II) We begin by observing that if $u \in \operatorname{supp} \psi_{t}^{x}$ and $v \in \operatorname{supp}\left\{\alpha^{x, t^{\prime}}-\alpha^{x, 2 t}\right\}$, then $|u-x|<\frac{1}{2}|x-v|$. We shall also use the estimate $\left|\psi_{t^{\prime}}^{y}(z)-\psi_{t^{\prime}}^{y}(x)\right| \leq C|z-x| / t^{\prime n+1}$. Thus again using the smoothness properties of $K, L$, and $M$,

$$
\begin{aligned}
\mid\left\langle\psi_{t}^{x},\right. & \left.K_{0}\left(\alpha^{x, t^{\prime}}-\alpha^{x, 2 t}\right)\left(\psi_{t^{\prime}}^{y}-\psi_{t^{\prime}}^{y}(x)\right)\right\rangle \mid \\
\leq & C \int\left|\psi_{t}^{x}(u)\right|\left[\frac{|x-u|^{\delta}}{|x-v|^{n+\delta}}\right] \frac{|x-v|}{t^{\prime n+1}} \cdot \alpha^{x, t^{\prime}}(v) d u d v \\
& +2 C \int\left|\psi_{t}^{x}(u)\right|\left[\frac{|x-u|}{|x-v|^{n+1}}\right] \frac{|x-v|}{t^{\prime n+1}} \cdot M_{10 t}, \beta^{\#}(v) \cdot \alpha^{x, t^{\prime}}(v) d u d v, \\
\leq & C \frac{(t)^{\delta}}{\left(t^{\prime}\right)^{n+\delta}} w_{p_{0}}^{-1}(x) .
\end{aligned}
$$

(III) In estimating the third quantity, we are forced to consider the weight $w_{p_{0}}$ as a function of $x$. We argue exactly as is done in [8].

Let $\left(t / t^{\prime}\right)^{n} \beta_{t}^{x}=\left(\psi_{t^{\prime}}^{y}-\psi_{t^{\prime}}^{y}(x)\right) \alpha^{x, 2 t}$. Then it is easy to show that the set of functions $\left\{\left(t^{\prime} / t\right) \beta\right\}$ are all supported in $\{|z|<40\}$, are $C^{\infty}$ and are uniformly bounded by $\|\nabla \psi\|_{\infty}$. By (4.2) we have

$$
\begin{aligned}
\left|\left\langle\psi_{t}^{x}, K_{0}\left(\psi_{t^{\prime}}^{y}-\psi_{t^{\prime}}^{y}(x)\right) \alpha^{x, 2 t}\right\rangle\right| & =\left(t / t^{\prime}\right)^{n+1}\left|\left\langle\psi_{t}^{x}, K_{0}\left(t^{\prime} / t\right) \beta_{t}^{x}\right\rangle\right|, \\
& \leq C \frac{t}{\left(t^{\prime}\right)^{n+1}} w_{p_{0}}^{-1}(x) .
\end{aligned}
$$

This completes the proof.

5. The $L^{2}$ theorem. Theorem 5.2 is the essential result of this paper in that the implications $(\S 6)$ follow by interpretation and the usual techniques. In our proof of this theorem, functions similar to the $S$ and $g_{\lambda}^{*}$ functions of [12] arise naturally. The $g_{\lambda}^{*}$ function is $L^{p}$ bounded for all $p \geq 2$ provided $\lambda>1$. For $1<p<2$, the $g_{\lambda}^{*}$ function is $L^{p}$ bounded for $p>2 / \lambda$, and here lies the problem in extending Theorem 5.2 for $p_{0}<2$.

For example, the function that we call $\tilde{g}_{\delta}(f)$ (see Definition 5.0) seems unavoidable in our proof of Theorem 5.2. This operator is $L^{p}$ bounded for $p \geq 2$, see Lemma 5.1 below, but the $L^{p}$ boundedness for $1<p<2$ will at least require $p>2 /((n+\delta) / n)$. Since $\delta$ is the same as in property $(1.1)$, it is interesting to note that there is some effect by assuming $0<\delta \leq 1$ rather than just 1 . Nevertheless we are only concerned with $p \geq 2$.

DEFINITION 5.0. Let $f \in C^{\infty}$ with compact support and $0<\delta \leq 1$. We define $S_{\psi} f$ and $\tilde{g}_{\delta} f$ for $x \in R^{n}$ as

$$
\begin{gathered}
S_{\psi} f(x)=\left|\int_{0}^{\infty} \int_{|x-y|<5 t}\left(Q_{t} f(y)\right)^{2} t^{-n-1} d y d t\right|^{1 / 2}, \\
\tilde{g}_{\delta} f(x)=\left|\int_{0}^{\infty} \int_{0}^{t} \int\left(Q_{t}, f(y)\right)^{2}\right| \frac{\left(t^{\prime}\right)^{\delta}}{t^{n+\delta}+|x-y|^{n+\delta}}\left|d y \frac{d t^{\prime}}{t^{\prime}} \frac{d t}{t}\right|^{1 / 2} .
\end{gathered}
$$

LEMMA 5.1. For $p \geq 2$,

$$
\left\|\tilde{g}_{\delta} f\right\|_{p} \leq C_{p, \delta}\|f\|_{p}, \quad\left\|S_{\psi} f\right\|_{p} \leq C_{p}\|f\|_{p}
$$

PROOF. The proof is basically that found in [12, Theorem 2, p. 91]. Similarly one observes $S_{\psi} f(x) \leq C_{\delta} \tilde{g}_{\delta} f(x)$.

We are now ready to prove the basic result. 
THEOREM 5.2. Let $K$ satisfy $\mathrm{WBP}_{0}(Q)$ for a $p_{0}>2$. Then there is a positive constant $C_{p_{0}}<1 / 400$ such that for $f$ in $L^{p}$ and supported in $C_{p_{0}} Q$ we have

$$
\begin{aligned}
& \int|K f(x)|^{2} w_{p_{0}}^{2}(x) d x \leq A_{p_{0}} \int|f(x)|^{2} d x, \\
& \int\left|K^{*} f(x)\right|^{2} w_{p_{0}}^{2}(x) d x \leq A_{p_{0}} \int|f(x)|^{2} d x,
\end{aligned}
$$

where

$$
w_{p_{0}}(x)=\left|\sup _{x \in J} \frac{1}{|J|} \int_{J}\left[\left|\beta_{L}^{\#}(s)\right|+\left|\beta_{M}^{\#}(s)\right|+1\right]^{p_{0}} d s\right|^{-1 / p_{0}}
$$

and

$$
\beta_{L}(x)=X_{Q}(x) \cdot K\left[X_{Q}\right](x)-\chi_{Q}(x) \operatorname{ave}_{Q} X_{Q} K\left[X_{Q}\right]
$$

and

$$
\beta_{M}(x)=X_{Q}(x) \cdot K^{*}\left[X_{Q}\right](x)-\chi_{Q}(x) \operatorname{ave}_{Q} X_{Q} K^{*}\left[X_{Q}\right] .
$$

PROOF. For notational ease we have assumed up until the statement of this theorem that the support of $f$ is contained in a cube $Q$ and for $Q_{0}=100 B_{2} Q$, $B_{2}>4$ (see Lemma 2.2), we have the operator $K$ satisfying $\mathrm{WBP}_{0}\left(Q_{0}\right)$, etc. So assume $f$ is supported in $Q$ and without loss of generality assume the center of $Q$ is 0 .

We break up the integration over $R^{n}$ into integrating over $R^{n} \backslash 4 Q$ and $4 Q$. Observe that (1.2) implies that $|K f(x)| \leq C[M f(x)]$, so with $w_{p_{0}}(x) \leq 1$ we have,

$$
\int_{R^{n} \backslash 4 Q}|K f(x)|^{2} w_{p_{0}}^{2}(x) d x \leq C \int|f(x)|^{2} d x .
$$

It is of course the integration over $4 Q$ that is interesting. To start we have $p_{0}>2$ so $\omega_{p_{0}}^{-2} \in A_{1}$. Observe that $w_{p_{0}}^{2} \in A_{2}$, so by $(2.3)$

$$
\begin{aligned}
\int_{4 Q}|K f(x)|^{2} w_{p_{0}}^{2}(x) d x \\
\leq B_{2} \int_{B_{2} Q}\left|\int_{0}^{B_{2} s(Q)}\left\{\iint Q_{t} K_{1} Q_{t},(x, y) Q_{t}, f(y) \frac{d t^{\prime}}{t^{\prime}} d y\right\}^{2} \frac{d t}{t}\right| w_{p_{0}}^{2}(x) d x \\
\quad+C_{2} \int|f(x)|^{2} d x,
\end{aligned}
$$

where the kernel of $K_{1}$ is given by $k(x, y) X_{Q_{0}}(x) X_{Q_{0}}(y)$. Using the decomposition of $K_{0}=K_{1}+L+M$, the first integral on the above right is bounded by

$$
\begin{aligned}
& 3^{2} \int\left[(L f(x))^{2}+(M f(x))^{2}\right] w_{p_{0}}^{2}(x) d x \\
& \quad+3^{2} \int_{B_{2} Q}\left|\int_{0}^{B_{2} s(Q)}\left\{\iint Q_{t} K_{0} Q_{t},(x, y) Q_{t}, f(y) \frac{d t^{\prime}}{t^{\prime}} d y\right\}^{2} \frac{d t}{t}\right| w_{p_{0}}^{2}(x) d x .
\end{aligned}
$$

By (3.2) and (3.3) the first integral is bounded by $C \int|f(x)|^{2} d x$.

We split up the last integral into three whose domains of integration in $y$ and $t^{\prime}$ correspond to that of (4.3), (4.4), and (4.5). 
For $|x-y|<5 t$ and $t^{\prime}<t$ we use (4.3) with Hölder's inequality to get

$$
\begin{gathered}
\left\{\int_{0}^{t} \int_{|x-y|<5 t} Q_{t} K_{0} Q_{t},(x, y) Q_{t}, f(y) d y \frac{d t^{\prime}}{t^{\prime}}\right\}^{2} \\
\leq C_{p_{0}}\left|\int_{0}^{t} \int_{|x-y|<5 t} \frac{w_{p_{0}}^{-2}(y)\left(t^{\prime}\right)^{\delta}}{t^{n+\delta}} d y \frac{d t^{\prime}}{t^{\prime}}\right|^{2} \frac{Q_{t^{\prime}}^{2} f(y)\left(t^{\prime}\right)^{\delta}}{t^{n+\delta}+|x-y|^{n+\delta}} d y \frac{d t^{\prime}}{t^{\prime}} \mid .
\end{gathered}
$$

Observe that the first factor is bounded by $C w_{p_{0}}^{-2}(x)$. If we now integrate (5.6) over $t$ and $x$ with respect to $w_{p_{0}}^{2}(x) d x d t / t$, the second factor becomes $\int\left[\tilde{g}_{\delta} f(x)\right]^{2} d x$ which by Lemma 5.1 is bounded by $C \int|f(x)|^{2} d x$.

The second domain of integration is $t<t^{\prime}$ and $|x-y|<5 t^{\prime}$. Note that the weighted estimate (4.4) has $w_{p_{0}}$ as a function of $x$. This simplifies matters as we can simply cancel out the weight $w_{p_{0}}$ and obtain the following integral

$$
\int_{B_{p} Q}\left|\int_{t}^{\infty} \int_{|x-y|<5 t^{\prime}} \frac{Q_{t^{\prime}}^{2} f(y)(t)^{\delta}}{\left(t^{\prime}\right)^{n+\delta}+|x-y|^{n+\delta}} \frac{d t}{t} \frac{d t^{\prime}}{t^{\prime}}\right| d x
$$

and changing the order of integration, one derives,

$$
\leq C \int\left[S_{\psi} f(x)\right]^{2} d x \leq C_{2} \int|f(x)|^{2} d x
$$

by Lemma 5.1 .

Finally in order to complete the proof we must consider the domain $|x-y|>$ $5 t \vee t^{\prime}$. Again we have that the weight $w_{p_{0}}$ in the estimate (4.5) is a function of $x$. Thus the weights cancel and we apply Hölder's inequality below,

$$
\begin{aligned}
&\left|\iint_{|x-y|>5 t \vee t^{\prime}} Q_{t} K_{0} Q_{t},(x, y) Q_{t}, f(y) \frac{d t^{\prime}}{t^{\prime}} d y\right|^{2} \\
& \leq {\left[\iint_{|x-y|>5 t \vee t^{\prime}} \frac{\left(\left.t \wedge t^{\prime}\right|^{\delta}\right.}{|x-y|^{n+\delta}+\left(t \vee t^{\prime}\right)^{n+\delta}} \frac{d t^{\prime}}{t^{\prime}} d y\right] } \\
& \cdot {\left[\int_{0}^{t} \int_{|x-y|>5 t} \frac{Q_{t^{\prime}}^{2} f(y)\left(t^{\prime}\right)^{\delta}}{|x-y|^{n+\delta}+t^{n+\delta}} d y \frac{d t^{\prime}}{t^{\prime}}\right.} \\
&+\left.\int_{t}^{\infty} \int_{|x-y|>5 t^{\prime}} \frac{Q_{t^{\prime}}^{2} f(y)(t)^{\delta}}{|x-y|^{n+\delta}+\left(t^{\prime}\right)^{n+\delta}} d y \frac{d t^{\prime}}{t^{\prime}}\right] .
\end{aligned}
$$

The first factor of (5.7) is easily computed to be a constant. When the right side of (5.7) is integrated over $t$ and $x$ with respect to $d x \cdot d t / t$, the first term of the second factor is easily seen to be dominated by $\left|\tilde{g}_{\delta} f\right|^{2}$. For the second term of the second factor, we first change the order of integration of $t$ and $t^{\prime}$. The integration of the variable $t$ can be carried out, then what is left is easily seen to be dominated by the standard $g^{*}$-function associated with $\psi$, i.e.,

$$
\left|g_{\psi}^{*} f(x)\right|^{2}=\int_{0}^{\infty} \int \frac{Q_{t^{\prime}}^{2} f(y)\left(t^{\prime}\right)^{\delta}}{|x-y|^{n+\delta}+\left(t^{\prime}\right)^{n+\delta}} d y \frac{d t^{\prime}}{t^{\prime}} .
$$


This is well known to be a bounded operator on $L^{2}$, so again with Lemma 5.1 we obtain $C \int|f(x)|^{2} d x$ as an upper bound completing the proof.

6. Applications. For our first application we will extend Theorem 5.2 to include other values of $p$ and to move the weight to the other side. From the operator $K$ there is an associated maximal singular operator $K_{*}$. We further extend our results to hold for $K_{*}$ rather than just $K$. Though $K_{*}$ is a stronger operator, it has nicer properties such as satisfying a good-lambda inequality. This inequality is such a nice tool when weights, other values of $p$, and so forth are involved that we would use the maximal operator in our proof even if we were only concerned with just $K$.

DEFinition 6.0. Given the operator $K$ and its adjoint $K^{*}$, we define the associated maximal operators as follows:

$$
\begin{aligned}
& K_{*} f(x)=\sup _{\varepsilon>0}\left|\int_{|x-y|>\varepsilon} k(x, y) f(y) d y\right|, \\
& K_{*}^{*} f(x)=\sup _{\varepsilon>0}\left|\int_{|x-y|>\varepsilon} k(y, x) f(y) d y\right| .
\end{aligned}
$$

Before we get to our next result let us save notation and so on by realizing that whatever is satisfied by $K$ and $K_{*}$ is also satisfied by the adjoint and its maximal operator.

THEOREM 6.1. Let $K$ satisfy $\mathrm{WBP}_{0}(Q)$ for a $p_{0}>2$. Then there is a positive constant $C_{p_{0}}<1 / 400$ such that for $f$ supported in $C_{p_{0}} Q$,

$$
\begin{aligned}
& \int\left[K_{*} f(x)\right]^{p} w_{p_{0}}^{p}(x) d x \leq A_{p_{0}, p} \int|f(x)|^{p} d x, \quad f \in L^{p}\left(C_{p_{0}} Q\right), \\
& \int\left[K_{*} f(x)\right]^{p} d x \leq B_{p_{0}, p} \int|f(x)|^{p} w_{p_{0}}^{-p}(x) d x, \quad f \in L_{w_{p_{0}}^{-p}}^{p}\left(C_{p_{0}} Q\right) .
\end{aligned}
$$

ProOF. The proof uses many standard techniques and so we will be brief in places and just provide an outline. However, there will be a spot where we shall again use the fact that $p_{0}>2$.

To begin, (5.4), (5.5), and duality imply

$$
\int|K f(x)|^{2}+\left|K^{*} f(x)\right|^{2} d x \leq B_{p_{0}} \int|f(x)|^{2} w_{p_{0}}^{-2}(x) d x .
$$

As has been mentioned we shall use maximal operators and to introduce them we need a result similar to Cotlar's inequality, [7]. That is, for $\varepsilon>0$, we have for $x \in C_{p_{0}} Q$,

$$
\left|\int_{|x-y|>\varepsilon} k(x, y) f(y) d y\right| \leq D_{p_{0}}(M K f(x))+\left(M\left[f^{2} w_{p_{0}}^{-2}\right](x)\right)^{1 / 2} .
$$

The proof of this is the same as the proof of Cotlar's inequality and uses (6.4). We note that (6.5) is true for the adjoint operator. 
Using (6.5) and the method of [2] the following good-lambda inequality can be established.

$$
\begin{aligned}
& \left|\left\{x \in R^{n}: K_{*} f(x)>(1+\varepsilon) \lambda,\left(M\left[f^{2} w_{p_{0}}^{-2}\right](x)\right)^{1 / 2} \leq C_{1} \varepsilon \lambda\right\}\right| \\
& \quad \leq C_{2} \varepsilon\left|\left\{x \in R^{n}: K_{*} f(x)>\lambda\right\}\right| .
\end{aligned}
$$

Again (6.6) also holds for $K_{*}^{*}$. Having (6.6) it is easy to get

$$
\int\left[K_{*} f(x)\right]^{p}+\left[K_{*}^{*} f(x)\right]^{p} d x C_{p_{0}, p} \int|f(x)|^{p} w_{p_{0}}^{-p}(x) d x
$$

for $2<p<\infty$.

Now we have extended (6.4) for $2 \leq p<\infty$ and arguing adjoints we have the statements of Theorem 5.2 true for $1<p \leq 2$. Continuing the proof, we wish to now show (6.4) for all $p$ near 1 , or equivalently, (5.4) and (5.5) for all large $p$. To do this we shall move the weight $w_{p_{0}}$ to the other side.

For $\varepsilon=\left(p_{0}-2\right) / 2$, we have $w_{p_{0}}^{-(2+\varepsilon)} \in A_{1} \subset A_{q}$ for all $q \geq 1$. Using the relationship $u \in A_{q}$ iff $u^{1-q^{\prime}} \in A_{q^{\prime}}, 1 / q+1 / q^{\prime}=1$, then $w_{p_{0}}^{p} \in A_{q^{\prime}}$, where $1-q^{\prime}=-p /(2+\varepsilon)$ or $q^{\prime}=1+p /(2+\varepsilon)$. In other words, $w_{p_{0}}^{p} \in A_{p / 2}$ for all $p / 2 \geq 1+p /(2+\varepsilon)$. This implies that the good-lambda inequality (6.6) is true for the weight $w_{p_{0}}^{p}$ for all sufficiently large $p$. From this it's easy to derive using distribution functions,

$$
\int\left[K_{*} f(x)\right]^{p}+\left[K_{*}^{*} f(x)\right]^{p} w_{p_{0}}^{p}(x) d x \leq D_{p_{0}, p} \int|f(x)|^{p} d x
$$

for all $p$ such that $p / 2 \geq 1+p /(2+\varepsilon)$. Again the adjoint argument gives us (6.4) for all $p$ sufficiently close to 1 , i.e., there is a $p_{2}$ such that for $1<p_{0}<p_{2}$,

$$
\int|K f(x)|^{p}+\left|K^{*} f(x)\right|^{p} d x \leq F_{p_{0}, p} \int|f(x)|^{p} w_{p_{0}}^{-p}(x) d x .
$$

With interpolation we have the statements of Theorem 6.1 true for $K$ and $K^{*}$ instead of the maximal operators. To complete the proof one may now use (6.9) to derive a sharper version of our Cotlar's inequality (6.5) using $\left(M\left[f^{p} w_{p_{0}}^{-p}\right](x)\right)^{1 / p}$ for all $p>1$, thus implying (6.7) for all $1<p<\infty$. This proves (6.3). To prove (6.2) for the maximal operator we do a variation of this by using (6.2) is true for $K$ and $K^{*}$ to establish another Cotlar-type inequality, i.e., the usual argument derives

$$
\left|K_{*} f(x)\right| \leq C_{p_{0}, p}\left(M_{w_{p}} K f(x)+\left\{M_{w_{p}}\left[f^{p}\right](x)\right\}^{1 / p}+M f(x)\right),
$$

where

$$
M_{w_{p}} g(x)=\sup _{x \in Q} \frac{1}{w_{p_{0}}^{p}(Q)} \int_{Q}|g(y)| w_{p_{0}}^{p}(y) d y .
$$

Note that since $w_{p_{0}}^{p}<1$ the $M f(x)$ term provides no difficulty. So we have $M_{w_{p}} K f$, $\left\{M_{w_{p}}\left[f^{p}\right]\right\}^{1 / p}$, and $M f$ map $L^{p}$ into wk- $L_{w_{p_{0}}^{p}}^{p}$ implying the same for $K_{*} f, 1<p<$ $\infty$. This completes the proof of $(6.2)$.

For our second application we consider a commutator with fractional differentiation defined in Murray [10]. Specifically, let $A$ be a scalar valued function of $R^{n}$. Consider the operator $C_{\delta} f$ defined as follows for $0<\delta<1$.

$$
\begin{aligned}
C_{\delta} f(x) & =\left[A,|D|^{\delta}\right] f(x) \\
& =c(\delta) \text { p.v. } \int \frac{[A(x)-A(y)]}{|x-y|^{1+\delta}} f(y) d y,
\end{aligned}
$$


where $|D|^{\delta}$ is fractional differentiation defined for tempered distributions $f \in$ $\mathscr{S}^{\prime}\left(R^{n}\right)$ as $\left(|D|^{\delta} f\right)^{\wedge}(t)=|t|^{\delta} f^{\sim}(t)$. This operator and the related Riesz potentials $I_{\delta}$ are discussed in Stein [12]. The normalizing constant can be easily computed as $c(\delta)=\pi^{\delta+n / 2} \cdot \Gamma(-\delta / 2) / \Gamma(n / 2-\delta / 2)$ using ideas in [12].

The kernel of $C_{\delta}$ is asymmetric and if $A$ belongs to $\operatorname{Lip}_{\delta}\left(R^{n}\right)$, that is

$$
\|A\|_{\delta}=\sup _{x, y} \frac{|A(x)-A(y)|}{|x-y|^{\delta}}<\infty,
$$

then the kernel is easily seen to satisfy (1.1) and (1.2). From Strichartz [14], we define $I_{\delta}(\mathrm{BMO})$ as the space of functions $f$ modulo constants that have $|D|^{\delta} f \in$ BMO. Murray [10] shows, for $n=1$, that $C_{\delta}$ is a bounded operator on $L^{2}$ if and only if $C_{\delta} 1=|D|^{\delta} A \in \mathrm{BMO}$, that is, $A$ belongs to $I_{\delta}(\mathrm{BMO})$. It should be noted that the T1 theorem of David and Journé [6] implies the same result for all $n$. In [14] it is shown that $I_{\delta}(\mathrm{BMO})$ is a proper subset of $\operatorname{Lip}_{\delta}\left(R^{n}\right)$ for $0<\delta<1$. It is for functions $A \in \operatorname{Lip}_{\delta}\left(R^{n}\right) \backslash I_{\delta}(\mathrm{BMO})$ that Theorem 6.1 becomes useful. We now state our result.

THEOREM 6.2. Let $A \in \operatorname{Lip}_{\delta}\left(R^{n}\right)$. Then for $1<p<\infty$, and $p_{0}>2$,

$$
\int C_{\delta, *}^{p} f(x) \cdot\left|M\left[\left(1+\left\{|D|^{\delta} A\right\}^{\#}\right)^{p_{0}}\right](x)\right|^{-p / p_{0}} d x \leq \int|f(x)|^{p} d x
$$

where $C_{\delta, *}$ is the associated maximal operator of (6.10).

PrOOF. Assuming the weight on the left side is not identically 0 , then this is easily seen to be a restatement of Theorem 6.1. Of course a similar inequality holds with the weight moved to the other side.

\section{REFERENCES}

1. A. P. Calderón, Cauchy integrals on Lipschitz curves and related operators, Proc. Nat. Acad. Sci. U.S.A. 74 (1977), 1324-1327.

2. R. Coifman and C. Fefferman, Weighted norm inequalities for maximal functions and singular integrals, Studia Math. 51 (1974), 241-250.

3. R. R. Coifman, A. McIntosh and Y. Meyer, L'intégrale de Cauchy definit un opérateur borné sur $L^{2}$ pour les courbes Lipschitziennes, Ann. of Math. (2) 116 (1982), 361-388.

4. R. R. Coifman and Y. Meyer, Au delà des opérateurs pseudo-différentiels, Astérisque No. 57.

5. R. R. Coifman and R. Rochberg, Another characterization of BMO, Proc. Amer. Math. Soc. 79 (1980), 249-254.

6. G. David and J.-L. Journé, A boundedness criterion for generalized Calderón-Zygmund operators, Ann. of Math. (2) 120 (1984), 371-397.

7. J.-L. Journé, Calderón-Zygmund operators, pseudo-differential operators and the Cauchy integral of Calderón, Lecture Notes in Math., vol. 994, Springer-Verlag, 1983.

8. __ Vector-valued singular integral operators and singular integral operators on product spaces, preprint.

9. B. Muckenhoupt, Weighted norm inequalities for the Hardy maximal function, Trans. Amer. Math. Soc. 165 (1972), 207-226.

10. Margaret A. M. Murray, Commutators with fractional differentiation and BMO Sobolev spaces, Indiana Univ. Math. J. 34 (1985), 205-215.

11. J. Rubio de Francia, Factorization and extrapolation of weights, Bull. Amer. Math. Soc. (N.S.) 7 (1982), 393-396. 
12. E. M. Stein, Singular integrals and differentiability properties of functions, Princeton Univ. Press, 1970.

13. E. M. Stein and G. Weiss, Fourier analysis on Euclidean spaces, Princeton Univ. Press, 1971.

14. R. S. Strichartz, Bounded mean oscillation and Sobolev spaces, Indiana Univ. Math. J. 29 (1980), 539-558.

15. R. L. Wheeden, On hypersingular integrals and Lebesgue spaces of differentiable functions. II, Trans. Amer. Math. Soc. 139 (1969), 37-53.

Department of Mathematics, Florida International University, UniverSITY PARK, MIAMI, FLORIDA 33199 